\title{
Stay in touch
}

\author{
The virtual world offers countless opportunities to interact with each other, yet, it remains \\ difficult to replace valuable in-person scientific discussions that often happen spontaneously \\ at a conference.
}

One year into this pandemic, many of us have become experts in zoom meetings, online conferences and virtual socials. Moving scientific exchange into the virtual world certainly has advantages. In a recent webinar and Q\&A session, which we hosted together with Nature Nanotechnology, we could welcome questions from researchers all over the world, while sitting in our living rooms that have been temporarily transformed into offices. Many new virtual seminar series have been launched over the past year by faculty and students, connecting researchers from different fields and areas, and helping us not only to stay connected but also to engage with communities, which we may otherwise not interact with.

However, ad hoc scientific discussions and unforeseen encounters which are often key to developing new ideas and projects, are difficult to recreate in an online setting. As Editors, we want to be part of the scientific community, learn about research projects and collaborations, engage in discussions and meet faculty and students. Interacting with the community is at the heart of our profession and really at the heart of science. We all hope that in-person meetings will become possible soon again; however, the amount of travelling we were used to in pre-pandemic times may remain limited, at least owing to the ongoing climate crisis.

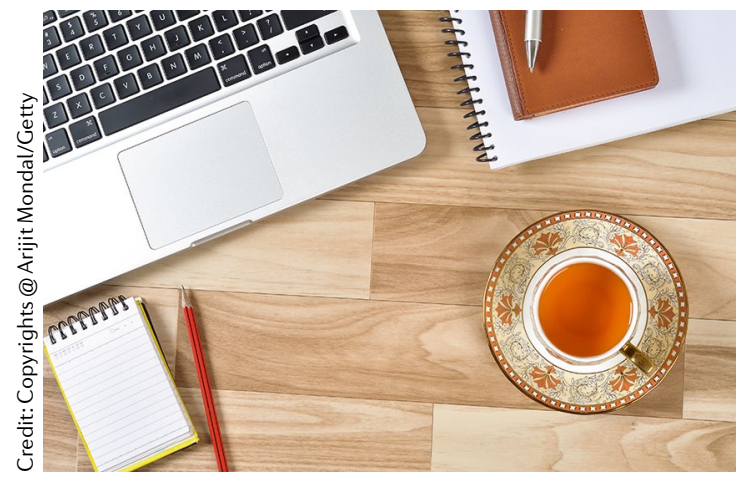

Therefore, we would like to take advantage of the connectivity of the virtual world, and we are excited about the opportunity to engage with researchers from various geographical areas. In the past year, we have participated in exciting student events, such as the Life Science Zurich Young Scientist Network to discuss editorial career opportunities with young researchers. We have given talks about scientific publishing to diverse audiences, including at a school hosted by the University of Sao Paulo in Brazil. We are keen to conduct virtual lab visits and virtual talks, and we also welcome ideas for webinars and Q\&A sessions, which we would be happy to host together with the community, perhaps as part of a seminar or conference series.

At Nature Reviews Materials, we further want to offer a platform, not only for Reviews and Perspectives but also for scientific discussion. Our Comment section provides the opportunity to discuss issues related to scientific policy, scientific community, translation and commercialization of materials, with the idea of reflecting ongoing discussions in the materials science community.

Our Comments cover a broad range of topics, from the state of clinical translation of nanoparticles for neural stimulation, the virtual community of Latinx researchers, LatinXinBME, 3D printing during the COVID-19 pandemic, how to find an academic position in materials science, the organization of an online conference, to a discussion about racism in academia, to name a few. The Comments should spark ideas, maybe even new collaborations, highlight new and established (online) communities within the materials science world and reflect on challenges within our community.

We want our journal to be the voice of our community, so please do reach out to us, and let us know if you would like to virtually meet, learn more about our journal or simply discuss scientific projects and articles. Let's stay in touch! 